\title{
Correct use of Membrane Elements in Structural Analysis
}

\author{
Timothy Rothman ${ }^{1}$ and Liming $\mathrm{Ji}^{2 \mathrm{a}}$ \\ ${ }^{1}$ Intel Corporation, 1900 Prairie City Road, Folsom, California, 95623, USA \\ ${ }^{2}$ Intel Corporation, Raycom Infotech Park A, No.2 KeXueYuan South Road, ZhongGuanCun, Beijing China 100190
}

\begin{abstract}
Structural analysis of consumer electronic devices such as phones and tablets involves Finite Element Analysis (FEA). Dynamic loading conditions such as device dropping and bending dictate accurate FEA models to reduce design risk in many areas. The solid elements typically used in structural analysis do not have integration points on the surface. The outer surface is of most interest because that is where the cracks start. Analysts employ a post processing trick through using membranes to bring accurate stress/strain results to the surface. This paper explains numerical issues with implementation of membranes and recommends a methodology for accurate structural analysis.
\end{abstract}

\section{Introduction}

Structural analysis of the complete system is done with different loadings such as bending or drop test [1]. Shear locking and hourglassing are usually understood and modelling techniques to avoid related errors are regularly used [2]. Finite Element Analysis (FEA) is used to solve this complex problem. During a typical design cycle at least 3 FEA iterations are needed. During a crash event, a number of structural components must sustain abnormal loadings in order to meet stringent integrity requirements [3]. Dynamic analysis to analyze drop test is not a trivial activity, and careful selection of element types and meshing strategies is needed. Membrane elements are used to accurately describe surfaces stresses related to underlying geometry that is not accurate at the surface when using solid elements alone [4]. These membrane elements need to be "glued" to the model, so *TIE or *EMBEDD [5] options are used. Careful use of membrane elements through mesh density, and method of attaching are explored.

\section{Structural analysis using FEA}

Structural analysis in the context of this paper is mechanical analysis of the complete system to different loadings such as bending or drop test [1]. Finite Element Analysis (FEA) or the Finite Element Method is used to discretize the domain and solve the 4 mathematical sets of equations of elasticity. It is usually necessary to characterize materials at appropriate loading rates to ensure the measured results will extend properly to the geometry of interest. In the design of phones, tablets and laptops correct structural design is of paramount concern and design cycles do not allow multiple

\footnotetext{
${ }^{\text {a }}$ Corresponding author : liming.ji@intel.com
} 
iterations. The philosophy is to make to the system correct by design the first time through mechanical analysis and allow iterations to be related to minor manufacturing or cosmetic issues. In a typical design cycle at least 3 design iterations are needed to work though design risk areas and ensure the proposed fixes are manufacturable and can be implemented in the limited physical space available for phones and tablets. Figure 1 show an example of structural design issues resolved using FEA.

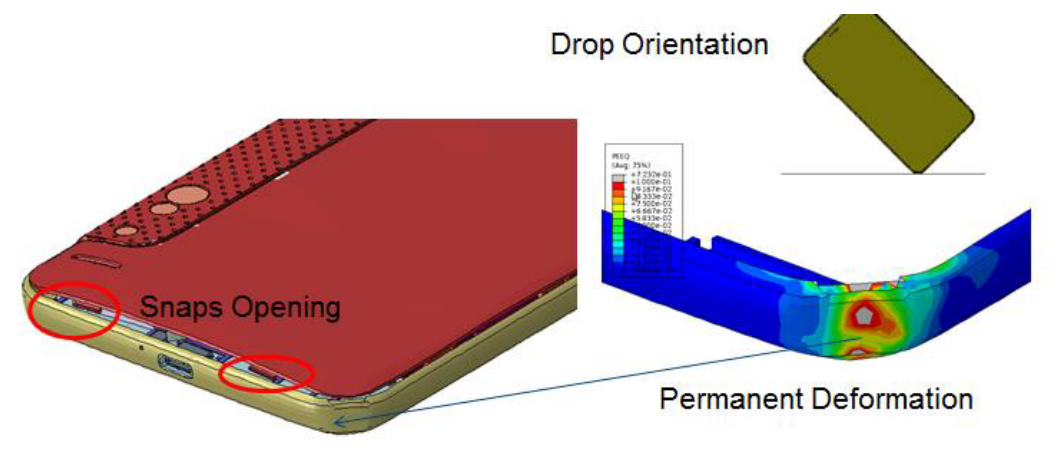

Figure 1. Drop Test at 1.5m FEA of prototype phone. Issues resolved during design process.

The major limitations to getting accurate, timely results are mesh accuracy, boundary or loading assumptions, run time relative to computational resources and schedule and material characterization accuracy. The focus of this paper is on the mesh accuracy. Mesh accuracy obviously includes how accurately the mesh matches the underlying geometry and if the mesh is too coarse, the area at risk may be missed entirely. Mesh accuracy also includes a much more subtle aspect of element choice, implementation and which elements to be used for which analysis.

\section{Element choices in Abaqus}

Commercial FEA tools like Simulia Abaqus provide analysts many choices of elements. The nodes are where the displacements are directly calculated, and the integration points are where additional information such as strains and stresses are calculated. Shown in Figure 2 are examples of 3D solid element each having 3 degrees of freedom (displacements) for each node. Many analysts rely on tribal knowledge on element choice due to the overwhelming choices available.
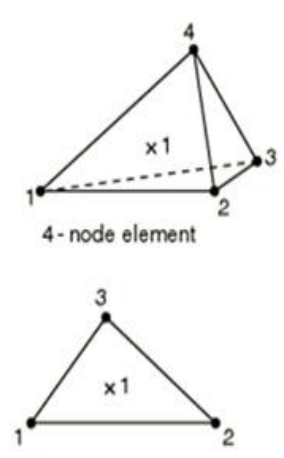

6- node element
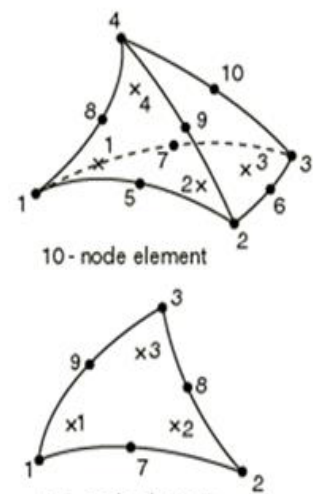

15- node element

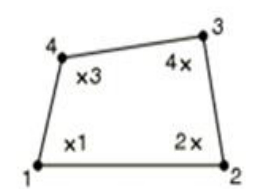

8- node element

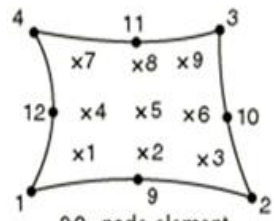

20-node element

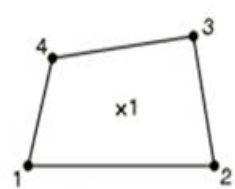

8 - node reduced integration element

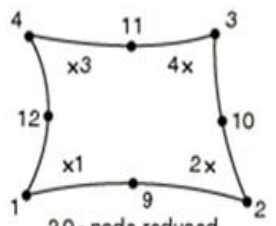

20- node reduced integration element

Figure 2. 3D Solid (Continuum) Element Types Available in Abaqus. x denotes integration point locations. 
There are many known sources of inaccuracy due to element density and choice. Element density is a fairly apparent source of error, but element choice is often misunderstood or the subject is avoided by the novice analyst. Poor choice of element density and type based on the geometry and loading often leads to erroneous results. In fact the true art of FEA lies in correct choice of the element.

\subsection{Shear locking and hourglassing}

It is well established that shear locking and hourglassing can dramatically affect the accuracy of the results [2]. Shear locking is observed with first order fully integrated elements in bending where the element mathematically cannot follow the actual shape caused by the loading. Many commercial FEA codes offer reduced integration element which is intended to resolve the shear locking phenomenon, while keeping computational time at a minimum. Hourglassing is the effect of a single integration point structure being excessively flexible unable to accurately represent the load handing ability of the structure. Even second order reduced integration elements suffer from hourglassing when only 1 layer of elements is used. Abaqus also provides in compatible mode elements that overcome the effects of shear locking and are practically not affected by hourglassing.

\subsection{Implementation of membrane elements}

The key structural components of the majority of transportation vehicles are designed as thin-walled components. During a crash event, a number of structural components must sustain abnormal loadings in order to meet stringent integrity requirements [3]. The loading and structural requirements for consumer electronics is similar, except transportation vehicles implement a "crush zone" and although the need for thin members is necessary to minimize consumer device thickness, the space for crush zones is typically not be available in handheld electronics.

Special element choices need to be considered for these high load, thin structures. The outer surface is of most interest because that is where the impact occurs and where the cracks start. The solid elements typically used in structural analysis do not have integration points on the surface (specifically C3D8I, C3D10M, and C3D10I). Analysts therefore use a post processing methodology to bring accurate stress/strain results to the surface. This is done by adding membrane elements to the model. [4] Membranes are used as the outer surface of every element in the model. The membrane in some cases is made directly from the outer faces of existing elements and no new nodes are created resulting in no new degrees of freedom and no additional run time needed for calculations (incremental run time need only for storing the results). In addition a small membrane called a patch is added for post processing in specific areas (i.e. such emulating a strain gage in an area of high solder stress under BGAs). These patches need to be "glued" to the model, so *TIE or *EMBEDD options are used.

\subsection{Case studies}

\subsubsection{Simple models}

In order to correctly assess accuracy models are simplified to ones which have analytical solutions. Figure 3 shows two simplified models chosen. One is a 200x40x1 $\mathrm{mm}$ beam in pure bending and held at one end (all degrees of freedom) and a moment applied at the free end. The second simplified model is a plate $100 \times 100 \times 1 \mathrm{~mm}$ bending due to uniform pressure held on 4 sides. Both models use Young's Modulus of $25 \mathrm{GPa}$ with poisson's ratio of 0.3 and element size of $0.5 \mathrm{~mm}$. 


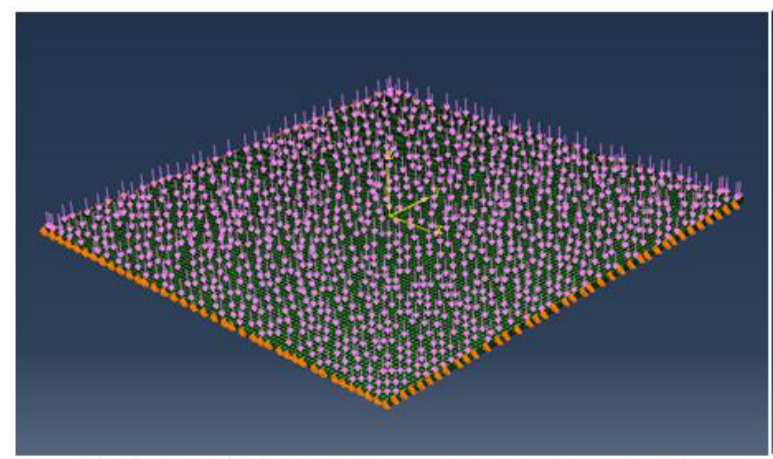

Simple Plate under pressure model: $100 * 100 * 1 \mathrm{~mm}$

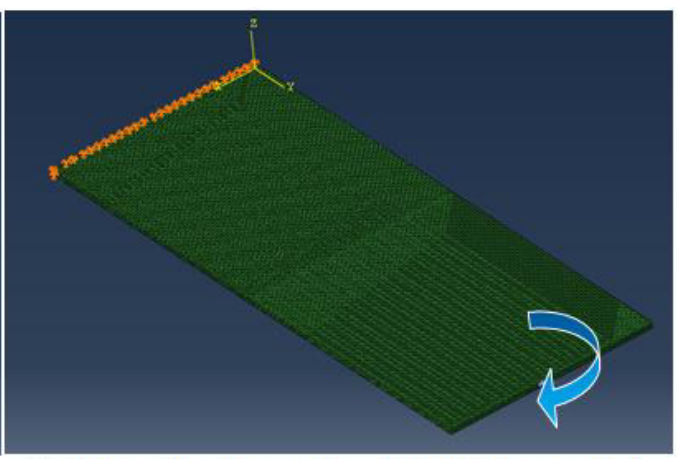

Simple Beam under bending model: $200 * 40 * 1 \mathrm{~mm}$

Figure 3. Simplified models. Orange denotes holding conditions.

Tables 1 and 2 indicate that the size of the membrane is NOT relevant to the results. Also the use of either *TIE or *EMBED has no impact on the patch used for post processing. There is only a minor influence of inaccuracy of the C3D10M element.

Table 1. Deformation and stress of Simple Plate under pressure indicating minimal inaccuracy using membranes.

\begin{tabular}{|c|c|c|c|c|}
\hline & Max def. U3 (mm) & Difference & $\begin{array}{c}\text { Stress (Mpa) S11 - Average } \\
\text { of int. pt. }\end{array}$ & Difference \\
\hline Analytical solution & 1.77200 & & 28.74000 & \\
\hline S4R & 1.7892 & $0.97 \%$ & 28.8948 & $0.54 \%$ \\
\hline C3D8I, 2 layers & 1.78497 & $0.73 \%$ & 28.8948 & $0.54 \%$ \\
\hline C3D8I, 2 layers + MEM & 1.78373 & $0.66 \%$ & 28.8266 & $0.30 \%$ \\
\hline C3D10M, one layer+MEM & 1.85727 & $4.81 \%$ & 30.0394 & $4.52 \%$ \\
\hline C3D10I, one layer+MEM & 1.78717 & $0.86 \%$ & 28.8608 & $0.42 \%$ \\
\hline C3D8I, 2 layer +MEM +0.8mm Patch -TIE & & & 28.8282 & $0.31 \%$ \\
\hline C3D8I, 2 layer +MEM +1.52mm Patch *TIE & & & 28.8215 & $0.28 \%$ \\
\hline C3D81, 2 layer $+\mathrm{MEM}+1.52 \mathrm{~mm}$ Patch *EMBED & & & 28.8173 & $0.27 \%$ \\
\hline
\end{tabular}

Table 2. Stress of Simple Beam under bending indicating minimal inaccuracy using membranes.

\begin{tabular}{|l|c|c|}
\hline & Curve - S22(Mpa) & Difference \\
\hline Analytical solution & $\mathbf{1 5}$ & \\
\hline C3D8I+MEM & 14.9909 & $-0.06 \%$ \\
\hline C3D8I, one layer+MEM & 14.991 & $-0.06 \%$ \\
\hline C3D8R+ENH+MEM & 14.9909 & $-0.06 \%$ \\
\hline C3D10I+MEM & 14.9905 & $-0.06 \%$ \\
\hline C3D10M + MEM & 15.5 & $3.33 \%$ \\
\hline C3D8I + 0.8mm Patch - *TIE & 14.997 & $-0.02 \%$ \\
\hline C3D8I + 0.8mm Patch - *EMBEDDED & 14.9975 & $-0.02 \%$ \\
\hline
\end{tabular}




\subsubsection{Complex models}

Simple models yield surprisingly accurate results, but the simplified state of bending can give a false sense of confidence because the same methodology a more complex model will give inaccurate results. In consumer electronics the BGA stress on the PCB is of key interest. Ensuring solder joint damage does not occur is critical. So a BGA package is added to a 100x60x1mm plate using C3D8I elements under pressure and patches are used in each BGA package corner. The stress plots in Figure 4 when using a $1.52 \mathrm{~mm} \times 1.52 \mathrm{~mm}$ patch seem similar between *TIE and *EMBEDDED, but closer inspection of the stress on the patches as shown in Table 3 suggests major differences. The $1.52 \mathrm{mmx} 1.52 \mathrm{~mm}$ patch is then discretized with $15 \times 15$ elements on each patch instead of a single element and Figure 5 and Table 4 show perfect correlation of results when using *TIE and *EMBEDDED.

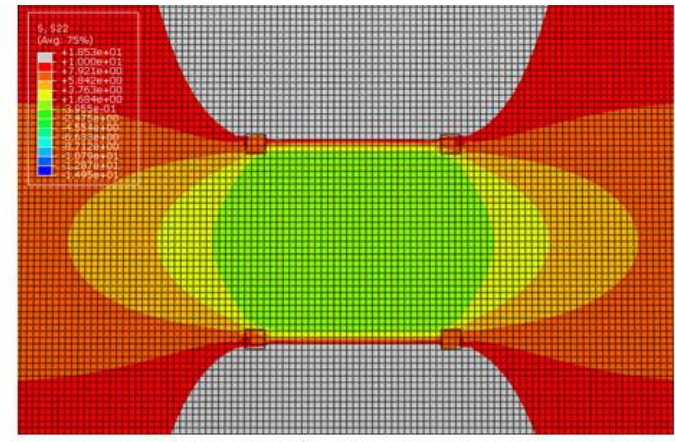

*TIE

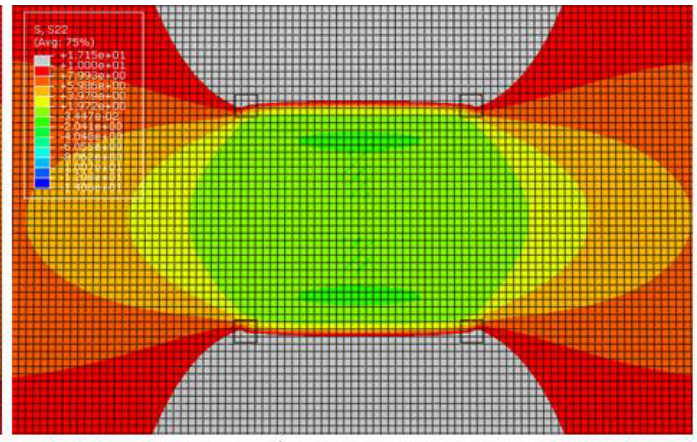

*EMBEDDED

Figure 4. Stress plots of complex model with BGA package using $1.52 \mathrm{mmx} 1.52 \mathrm{~mm}$ patch shown on the same scale suggesting the stress fields are the same

Table 3. Stress comparison of complex model with BGA package on $1.52 \mathrm{mmx} 1.52 \mathrm{~mm}$ patch. ${ }^{*} \mathrm{TIE}$ and *EMBEDDED do NOT match.

\begin{tabular}{|c|c|c|}
\hline Corner & *TIE & *EMBEDDED \\
\hline 1 & 7.67974 & 9.08999 \\
\hline 2 & 7.66387 & 9.08827 \\
\hline 3 & -7.60217 & -9.84414 \\
\hline 4 & -7.60342 & -9.84611 \\
\hline
\end{tabular}

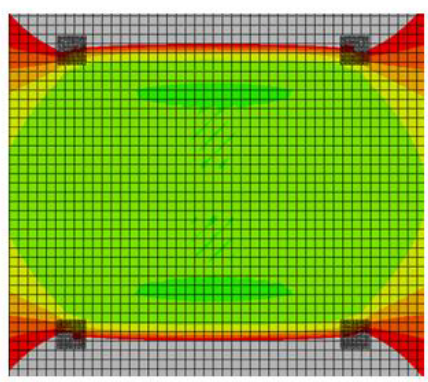

Patch size: $1.52 \mathrm{~mm} * 1.52 \mathrm{~mm}$

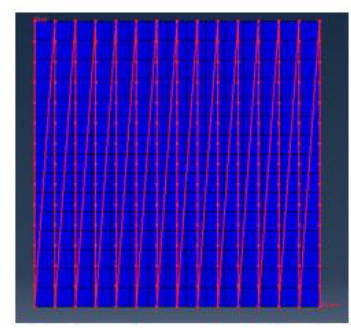

Zoomed view of patch 15*15 Elements

Figure 5. Stress plots of complex model with BGA package using $1.52 \mathrm{mmx} 1.52 \mathrm{~mm}$ patch discretized 
Table 4. Stress comparison of complex model with BGA package on $1.52 \mathrm{mmx} 1.52 \mathrm{~mm}$ patch discretized with $15 \times 15$ elements. ${ }^{*}$ TIE and $*$ EMBEDDED are in agreement.

\begin{tabular}{|c|c|c|}
\hline Corner & *TIE & *EMBEDDED \\
\hline 1 & 9.60765 & 9.60726 \\
\hline 2 & 9.59861 & 9.5982 \\
\hline 3 & -9.98875 & -9.98875 \\
\hline 4 & -9.99104 & -9.99104 \\
\hline
\end{tabular}

Cautionary note for using tetrahedral elements such as C3D10M. The C3D10M has middle nodes and in cases such as shown in Figure 1 where the corresponding membrane is M3D3 used as a coating on the underlying tetrahedral, the M3D3 may not yield accurate results since it has no midside nodes.

\section{Conclusions}

Care must be taken in ensuring the membrane skin or local post processing patch are the correct size and element type to match the underlying element bending behaviour. An accurate mesh is needed for both the solid and the membrane and the membrane should be used to bring the correct results to the surface, not create a new set of inaccurate results. If the membrane is bigger than the under solid element, the output may not be correct in bending for both *TIE and *EMBEDDED, the under beam is curved but big membrane element cant capture this deflection. See Figure 6.

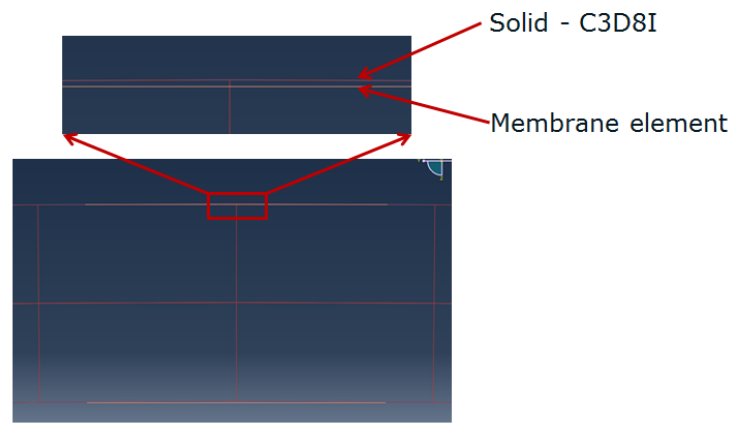

Figure 6. Exploded view of Membrane element placed just below surface of underling solid

\section{References}

1. JEDEC Standard JESD22-B111, Board level drop test method of components for handheld electronic products (2003)

2. Eric Quili Sun, Shear Locking and Hourglassing in MSC Nastan, ABAQUS, and ANSYS, MSC.Software's Virtual Product Development Conference 2006 Web Publication (2006)

3. T. Deihl , D. Carroll, Utilizing ABAQUS' 10-Node Modified Tet for Analyzing Impact Problems Involving Thin-Walled Structures, Proceedings Abaqus Users' Conference 2000, Newport RI (2000)

4. W. Abramowicz, Thin-walled Structures as Impact Energy Absorbers, Thin-Walled Structures 91-107 (2000)

5. Abaqus Analysis User's Guide

\section{Appendix}


*EMBEDDED - Abaqus searches for the geometric relationships between nodes of the embedded elements and the host elements. If a node of an embedded element lies within a host element, the translational degrees of freedom at the node are eliminated and the node becomes an "embedded node." The translational degrees of freedom of the embedded node are constrained to the interpolated values of the corresponding degrees of freedom of the host element. Embedded elements are allowed to have rotational degrees of freedom, but these rotations are not constrained by the embedding [5].

*TIE - Constrains each of the nodes on the slave surface to have the same motion as the point on the master surface to which it is closest [5].

Both TIE and EMBEDDED may get incorrect result if the membrane patch is much bigger than the under solid element. 